\title{
Ocular Cicatricial Pemphigoid: Camouflaged as Persistent Conjunctivitis
}

\author{
Jamal-uddin Mughal1, Asif Jan Muhammad2 and Uzma Erum²
}

\begin{abstract}
Ocular cicatricial pemphigoid (OCP) is a rare autoimmune disease with heterogenous clinical manifestations. The classical lesion involves ocular conjunctiva that leads to progressive scarring and fibrosis which ultimately results in visual loss. We report a case of a young male, who had chronic conjunctivitis which remained un-responsive to conventional treatment and results in significant morbidity. Later, it was diagnosed as ocular cicatricial pemphigoid involving eyes, nasal cavity, larynx as well as skin. Patient received immuo-suppressive therapy, which helped in arresting the disease process.
\end{abstract}

Key Words: Mucous membrane pemphigoid, Chronic conjunctivitis, Immunomodulation, Cicatricial pemphigoid.

\section{INTRODUCTION}

Ocular cicatricial pemphigoid (OCP), commonly referred as mucous membrane pemphigoid (MMP), is a rare, autoimmune disease with multi-system involvement. It usually affects elderly but adolescents may develop the disease with severe clinical manifestations. The reported incidence varies from $0.8-2$ per million population per year. ${ }^{1,2}$

The hallmark of the disease is cicatrizing conjunctivitis, however, variable involvement of other systems such as, oral and nasal cavity, genital area, larynx, pharynx, esophagus, and skin may occur. ${ }^{3}$ In fact, involvement of trachea and esophagus is potentially life-threatening. Exact pathogenesis of the disease remains to be elucidated but immune mediated type-II hypersensitivity has been thought to be responsible for the initiation of disease process. Clinical sequale include erosions, bullae formation, and progressive fibrosis leading to scarring. Untreated cases ultimately results in sight loss, due to blinding keratopathy. Thus, an early diagnosis and timely intervention is the key to cure. Here, we report a case of young male who had predominant ocular symptoms that were attributed to chronic conjunctivitis; and later it was diagnosed as OCP involving eyes, nasal cavity, larynx as well as skin.

We aimed to develop awareness among healthcare professionals, to impose a high index of suspicion in patients with non-resolving conjunctivitis, not only for the adequacy of diagnosis but also to facilitate early treatment and to prevent devastating sequale.

Department of Ophthalmologyl / Medicine ${ }^{2}$, Patel Hospital, Gulshan-e-Iqbal, Karachi, Pakistan

Correspondence: Dr. Uzma Erum, Department of Medicine,

Patel Hospital, ST-18, Block-4, Gulshan-e-Iqbal, Karachi,

Pakistan

E-mail:drkhan8583@gmail.com

Received: June 23, 2018; Accepted: November 27, 2018

\section{CASE REPORT}

A 37-year man, who had been living in Dubai for the last 10 years, driver by profession, first presented in December 2017 with complaint of redness of eyes, mucous discharge from eyes, visual impairment, hoarseness of voice and nasal stuffiness for eight months. Visual loss was gradual and it had led to complete loss of vision due to corneal perforation in the left eye, and reduced visual acuity in the right eye. There was history of recurrent conjunctivitis, grittiness in eyes and recurrent oral ulceration. Change in voice was noted for the last six months; initially, it became husky, and later his sound production was almost lost, though he was able to speak. He also had multiple, non-itchy, hyper-pigmented, maculopapular lesions all over body. There was nasal congestion with post-nasal drip, but no foul smell. There was history of $20-\mathrm{Kg}$ weight loss in the preceding six months. There was no history of ocular trauma as well as no complaint of photosensitivity, malar rash, alopecia, dysphagia, backache, arthralgia, genital ulceration or uretheral discharge.

Examination showed a young man of good built, BMI $27.6 \mathrm{Kg} / \mathrm{m}^{2}$. There was red, congested conjunctiva, with symblephara and shortening of fornix with limited EOM. A corneal flap was also visible in left eye. Visual acuity in right eye was $6 / 36$ and it was $6 / 60$ in the left eye. Voice was hoarse; there was no goiter. Fiber optic laryngoscopy (FOL) showed laryngeal edema, with narrow laryngeal inlet and crusting over vocal cords. There was deviation of nasal septum and crusting over turbinates. In addition, there were multiple, post-inflammatory hyperpigmented, macular lesions over chest, trunk and back. Oral mucosa was normal. Systemic examination was unremarkable.

Initially a diagnosis of Behcet's disease was considered, but neither it fit into the criteria nor pathergy phenomenon was demonstrated. His laboratory work-up was negative for any systemic or autoimmune rheumatic disease. His $\mathrm{Hb}$ was $14.4 / \mathrm{dl}$, WBC $8.8^{*} 10^{9} / \mathrm{L}$ (PMN - 


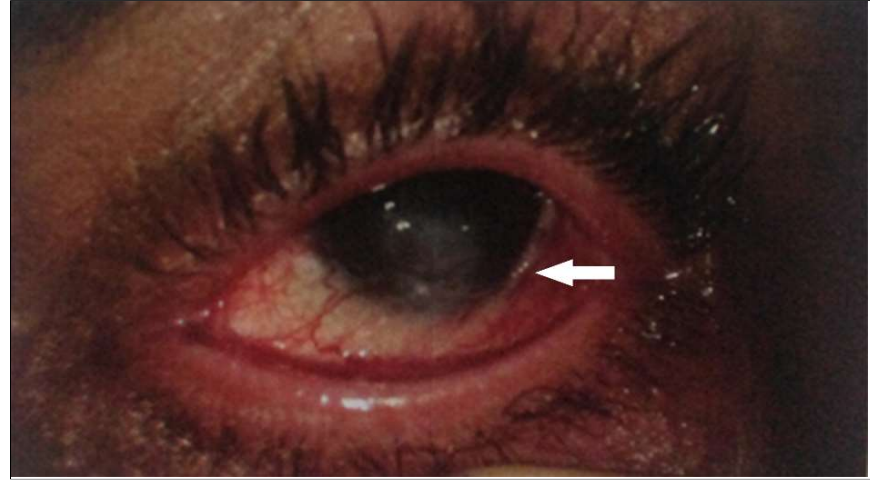

Figure 1a: Symblephara

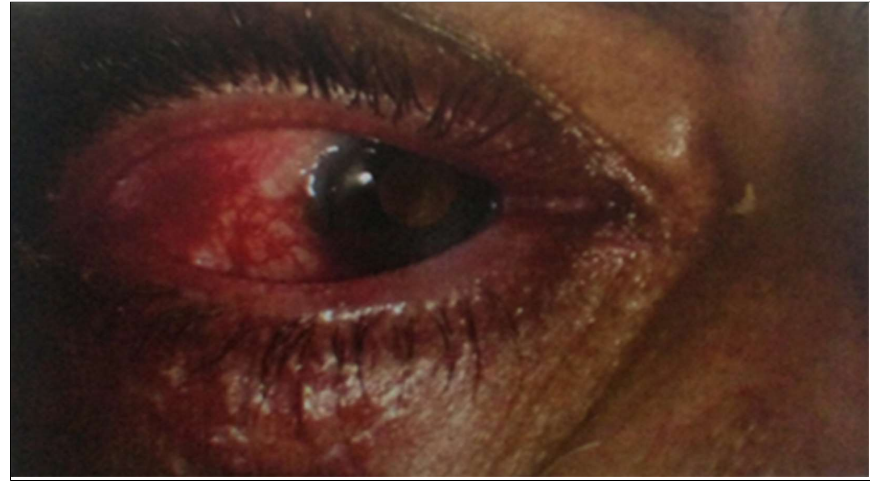

Figure 1b: Leucoma and corectopia.

$80 \%$, lymphocytes $14 \%$ ), platelets $275^{*} 10^{\circ} /$ L, ESR was $69 \mathrm{~mm} / \mathrm{Hr}$ and CRP $12.2 \mathrm{mg} / \mathrm{dl}$. Renal functions and coagulation profile were normal, autoimmune workup including, ANA, anti-DsDNA, pANCA, cANCA, ENA profile was also negative. Considering Wegener's granulomatosus as another differential his nasal turbinate bipsy was done, which showed squamous slough and nonspecific inflammatory exhudates with no evidence of granuloma or malignancy. His throat swab and nasal scrapings for culture and sensitivity, gram stain and fungal smear were all negative. CT-scan neck and paranasal sinuses was also normal.

Topical eye treatment was given in the form of lubricant drops and tobramycin. He developed corneal perforation, flap repair was done; but his symptoms continued. Ophthalmology review was taken again which demonstrated the presence of conjunctivial congestion, severe dry eyes, symblephara (adhesion between the palpebral conjunctivia of eyelid and bulbar conjunctivia of the eye ball), ankyloblepharon (fusion of all or part of eyelid margins), and fornix shortening (Figures 1a-1b). There was a small corneal perforation in left eye with adherent leucoma (corneal opacity) formation and uneven anterior chamber and corectopia (displacement of pupil from its normal central position) that helped in the diagnosis of OCP. Conjuctival biopsy was deferred due to increased risk of scarring, and clear diagnosis on ophthalmoscopic examination.
Due to his progressive visual symptoms, six cycles of cyclophosphamide $500 \mathrm{mg}$ were given at two-weekly intervals, with high dose oral steroids (Prednisolne $1 \mathrm{mg} / \mathrm{Kg} / \mathrm{d}$ ). Patient gradually improved in terms of clinical symptoms. Redness in eyes and photophobia has markedly settled now. Visual acuity in right eye is $6 / 18$, with residual pin-hole vision (6/60) in the left eye. The quality of voice was improved and he was able to produce sound. Repeat FOL showed $80 \%$ reduction in crusting over his vocal cords. Patient was compliant to regular follow-up and was on maintenance steroids (prednisolone) and azathioprine 100mg per day.

\section{DISCUSSION}

MMP or OCP has varied clinical manifestations and its heterogenous presentation imposes diagnostic challenge. The success of treatment depends upon an early and prompt diagnosis. Hence, it is appropriate to state that early diagnosis is the paradigm to cure OCP.

The recalcitrant ocular manifestation in this case of MMP should have been recognised earlier, before considerable damage to sight had occurred. Diagnosis for OCP is based on clinical as well as positive direct immunofloursence (DIF) from peri-lesional tissue, that demonstrates the deposition of immune-reactants at the epithelial basement membrane. ${ }^{3}$ The ocular findings are distinct towards the diagnosis of cicatrizing pemphigoid, based on Foster's classification for the diagnosis and staging of OCP. However, the current staging systems for OCP are based on subjective assessment of conjunctival fibrosis and fornix shortening and is dependent on an individual's interpretation, so both clinical and histolgical parameters are required to confirm the diagnosis. It has been reported that if the ocular findings are suggestive of OCP, then biopsy is not mandatory for the diagnosis, as the DIF of the tissue sample may remain inconclusive and it does not distinguish OCP from other blistering diseases such as bullous pemphigoid, epidermolysis bullosa and bullous lupus. ${ }^{4}$ It has been suggested that, nearly all cases of OCP or MCP with active disease require systemic immune-modulator treatment to suppress inflammation, regardless of the biopsy results. Furthermore, it should be kept in mind that bulbar conjunctival biopsy may lead to further worsening of scarring. In addition, we did all the necessary autoimmune works to establish the underlying cause for this patient's clinical mani-festations, which remained negative. This patient had corneal flap repair, but surgical management per se is quite challenging in scarred tissue. It has been said that whenever there is little conjunctival reserve, surgical interventions are difficult and usually result in high failure rate.

For cases with the diagnosis of OCP, the treatment of choice varies with the site of involvement. Patients with 
sight-threatening ocular involvement are best treated with cyclophosphamide (CYP). As this patient had profound ocular as well as laryngeal involvement, CYP was used as first line drug to induce remission. Conventional immunosuppressant drugs such as mycophenolate mofetil, azathioprine, cyclosporin are also considered a cornerstone to impede the autoimmune disease process in the management of OCP. 5 Dapsone and methotrexate have also been considered a reasonable option to treat mild cases of MMP. In addition to this, recent evidence suggests that rituximab (RTX) is a reasonable option with proven effectivness in limiting the disease progression. ${ }^{6}$ Also, novel regenerative techniques such as stem cell therapy might be considered for restoration of vision in patients with significant corneal scarring. ${ }^{7}$ Furthermore, the combination of RTX and intravenous immunoglobulin has also been reported to arrest the progression of disease and to prevent devastating sequale.8,9 RTX was reserved for any further worsening of symptoms or if patient might have any disease flare in near future. In addition, TNF- $\alpha$ inhibitors may play substantial role in the management of OCP as TNF- $\alpha$ appears to have pro-inflammatory and pro-fibrotic effects on conjunctival fibroblasts. Nonetheless, several gaps exist in the understanding of how optimally patient could get the benefit of long term immunosuppression that has been given to the patient.

Close follow-up is imperative for disease progression, as studies have reported an increased recurrence of OCP after discontinuation of treatment. It has been reported that long term disease suppression can be achieved with implementation of immune-modulator therapy in stepladder pattern. Also, combination of both topical and systemic immunomodulator treatment has a superior role to control active disease progression in cases of severe ocular, laryngeal or multi-system involvement. A Europeon study reported relapse in $22 \%$ of patients after successful remission. 10 However, no specific predictors of prognostic significance for relapse have been reported. In general, prognosis is better in patients where the disease is limited to oral mucosa, while ocular involvement is nearly always face treatment resistance or relapse at later time. Persistent epithelial defects and ongoing conjunctival inflammation lead to keratopathy and visual handicap. Abrupt cessation of treatment results in severe disease relapse and sometimes worsening of clinical symptoms ensue very rapidly.

So far, this patient has shown prompt response to Cyp arresting the disease process at this point. Nonetheless, it is too early to predict that how well the patient would maintain this response in future and how long the autoimmune disease process would remain suppressed with current treatment regimen. Furthermore, this patient had a significantly longer duration of disease, before immune-suppression was started; hence, the maintenance of remission might need prolonged treatment duration. The disease is rare, and most studies pertaining to MMP or OCP have been limited to individual case reports or case series; long term treatment response is largely unknown. Accurate identification of the disease at an early stage pose difficulty, as it is largely dependent on subjective assessment. The authors propose to develop a uniform rationale while treating patients with relapsing or persistent chronic conjunctivitis, so that an early diagnosis would be made and prompt and appropriate treatment should be instituted at appropriate time to manage the devastating consequences of the disease.

\section{REFERENCES}

1. Radford CF, Rauz S, Williams GP, Saw VP, Dart JK. Incidence, presenting features and diagnosis of cicatrizing conjunctivitis in the United Kingdom. Eye (Lond) 2012; 26:1199-208.

2. Bertram F, Brocker EB, Zilikens D, Schmidt E. Prospective analysis of the incidence of autoimmune disorders in Lower Franconia, Germany. J Dtsch Dermatol Ges 2009; 7:434-40.

3. Chan LS, Ahmed AR, Anhalt GJ, Bermauer W, Cooper KD, Elder MJ, et al. The first international consensus on mucous membrane pemphigoid: definition, diagnostic criteria, pathogenic factors, medical treatment and prognostic indicators. Arch Dermatol 2002; 138:370-9.

4. Williams GP, Radford C, Nightingale P, Dart JKG, Rauz S. Evaluation of early and late presentation of patients with ocular mucous membrane pemphigoid to two major tertiary care hospitals in the United Kingdom. Eye 2011; 25:1207-18.

5. Saw VP, Dart JK, Rauz S, Ramsay A, Bunce C, Xing W, et al. Immunosuppressive therapy for ocular mucous membrane pemphigoid strategies and outcomes. Opthalmology 2008; 115:253-61.

6. You C, Lamba N, Lasave AF, MaL, Diaz MH, Foster CS. Rituximab in the treatment of ocular cicatricial pemphigoid: A retrospective cohort study. Graefes Arch Clin Exp Opthalmol 2017; 255:1221-8.

7. Srikumaran D, Akpek EK. Mucous membrane pemphigoid: Recent advances. Curr Opin Opthalmol 2012; 23:523-7.

8. Queisi MM, Zein M, Lamba N, Meese H, Foster CS. Update on ocular cicatricial pemphigoid and emerging treatments. Surv Opthalmol 2016; 61:314-7.

9. Steger B, Madhusudan S, Kaye SB, Stylianides A, Romano V, Maqsood SE, et al. Combined use of Rituximab and intravenous immunoglobulin for severe autoimmune cicatricial conjunctivitis - An interventional case series. Cornea 2016; 35 : 1611-4.

10. You C, Ma L, Anesi SD, Stephen Foster C. Long term remission of ocular cicatricial pemphigoid off immunomodulatory therapy. Eur J Opthalmol 2018; 28:157-62. 